\title{
Investigation and Analysis of Iron-Deficiency Anemia Complicated by Splenomegaly
}

\author{
Yan-hui Wei (1D) \\ Yu-zhuo $\mathrm{He}^{2}$ \\ Xiao-yan Guo (D) \\ Xiao-yan Lin $^{2}$ \\ Hong-bin Zhu ${ }^{2}$ \\ Xue-jun Guo ${ }^{2}$
}

'Department of Graduate School, Xinxiang Medical University, Xinxiang, Henan Province, 453003, People's Republic of China; ${ }^{2}$ Department of Hematology, Puyang Oilfield General Hospital, Puyang, Henan Province, 457000, People's Republic of China
Correspondence: Xue-jun Guo

Department of Hematology, Puyang Oilfield General Hospital, Puyang, Henan Province, 457000, People's Republic of China $\mathrm{Tel} / \mathrm{Fax}+86-139039306 / 2$

Email pygxj@163.com
Objective: This study aimed to determine the incidence of iron-deficiency anemia (IDA) complicated by splenomegaly in our hospital over the past 6 years and to analyze the possible causes of this result.

Methods: This is a retrospective study. In total, 668 patients with IDA who were hospitalized in the hematology department of our hospital from 2013 to 2019 were selected as the research subjects and included in the IDA group, and 3201 patients who underwent outpatient physical examinations in our hospital during the same period were included in the control group. The incidences of splenomegaly in the IDA and control groups were calculated, and the difference was analyzed by means of statistical methods.

Results: Among the 668 IDA patients, 46 (6.9\%) had splenomegaly, and among the 3201 patients in the control group, 21 had splenomegaly $(0.7 \%)$. The incidence of splenomegaly was significantly higher in the IDA group than in the control group, and the severity of anemia in the IDA group was associated with the occurrence of splenomegaly. Specifically, the incidence of splenomegaly was $12.4 \%$ among patients with severe anemia and as high as $50 \%$ among patients with extremely severe anemia.

Conclusion: IDA is correlated with the incidence of splenomegaly, and the incidence of splenomegaly significantly increases as the severity of IDA increases. This is considered to be caused by extramedullary hematopoiesis.

Keywords: iron-deficiency anemia, splenomegaly, extramedullary hematopoiesis, cytokines

\section{Introduction}

Iron-deficiency anemia (IDA) refers to microcytic hypochromic anemia, a condition most commonly caused by insufficient levels of iron in the body, resulting in reduced hemoglobin synthesis. ${ }^{1-3}$ The World Health Organization estimates that IDA has affected $35 \%$ of the global population and up to $50 \%$ of pregnant women worldwide; ${ }^{2}$ notably, the prevalence of IDA among children in some underdeveloped areas may be higher than $50 \%{ }^{1,3}$ The spleen, which is the largest organ involved in the immunological functions, carries out some hematopoietic functions during early embryonic development. However, the hematopoietic function of the spleen basically disappears after birth, and instead, the spleen becomes the center of cellular and humoral immunity, playing a role in removing aging blood cells (such as red blood cells), antigens and foreign bodies and stimulating immune responses. ${ }^{4-6}$ Additionally, in some pathological contexts (eg, a severe hematopoietic disorder in the human body), the spleen can be stimulated to restore hematopoietic function, and restoration of this function is often accompanied by splenomegaly. Splenomegaly is a clinical manifestation of systemic diseases and 
is most commonly seen in hematologic diseases such as leukemia, lymphoma, and idiopathic thrombocytopenic purpura (ITP). However, in clinical practice, it has been found that some patients with IDA have concomitant splenomegaly. This study analyzes the correlation between IDA and splenomegaly and investigates the causes of IDA with splenomegaly. The findings of this study are expected to contribute to the differential diagnosis of splenomegaly, assist clinicians in making a correct diagnosis of patients with splenomegaly in clinical practice, and provide ideas for relevant research in the future.

\section{Data and Methods}

\section{General Information}

Patients with complete clinical data who were seen in the Hematology Department of Puyang Oilfield General Hospital from 2013 to 2019 were selected as the research subjects. Patients were diagnosed with microcytic hypochromic anemia based on laboratory-based measurements of the hemoglobin concentration, average volume of red blood cells, and average concentration of red blood cell hemoglobin. Then, as the subjects chosen for the preliminary analysis, patients with IDA underwent evaluations of ferritin levels, transferrin levels, transferrin saturation, serum iron levels and bone marrow biopsy. The examination records of the patients, including records of liver, gallbladder, spleen, pancreas and kidney ultrasound, tumor marker evaluations, and gastroenteroscopy procedures, were consulted to determine whether there were other secondary causes that might lead to splenomegaly in these patients. A total of 668 patients with IDA (117 males and 551 females) were identified as the final study subjects. A total of 3201 patients (1530 males and 1671 females) from the normal population who underwent physical examinations in our hospital during the same period were selected for the control group. The exclusion criteria were as follows: After the discovery of splenomegaly, relevant tests were performed to check and exclude patients with splenomegaly caused by other diseases from the experimental and control groups (eg, cirrhosis of the liver, blood diseases, parasitic infections, tumors, etc.).

\section{Instruments and Methods}

Routine examinations of the liver, gallbladder, spleen, and pancreas were performed with a Vivid E9 diagnostic ultrasound system (GE, USA). A spleen thickness greater than
$4 \mathrm{~cm}$, a longest spleen diameter $>11 \mathrm{~cm}$, and a positive physical exam were used as the criteria for confirmed splenomegaly. ${ }^{7,8}$ The data on sex and disease prognosis of the patients in the experimental group were collected.

\section{Statistical Methods}

Statistical analysis was performed by means of SPSS 21.0 software. The chi-squared test was performed to compare differences between groups. $\mathrm{P}<0.05$ indicated that a difference was statistically significant.

\section{Results}

The comparison of the incidences of splenomegaly between the IDA group and the control group is presented in Table 1. Among the 668 IDA patients, 46 had splenomegaly, yielding an incidence of $6.4 \%$. Among the 3201 control subjects, 21 had splenomegaly, yielding an incidence of $0.7 \%$. The difference in the incidence of splenomegaly between the IDA and control groups was significant $(\chi 2=126.059, \mathrm{P}<0.05)$.

The comparison of the incidence of splenomegaly among patients with different degrees of IDA is presented in Table 2. Among the 668 IDA patients, 3, 16, 24, and 3 in the mild, moderate, severe, and extremely severe anemia groups, respectively, had splenomegaly. When we analyzed the incidence of splenomegaly in these anemia groups, we found a significant difference between the four groups $\left(\chi^{2}=23.889\right.$, $\mathrm{P}<0.05)$. The incidence of splenomegaly in the severe anemia group was significantly higher than that in the mild or moderate anemia group. Furthermore, the extremely severe anemia group had a significantly higher incidence than the above three groups.

Follow-up observations were conducted one year ago. Among the 46 patients with IDA and splenomegaly, four had died (one died in an accident, two died of cerebral

Table I Comparison of the Incidence of Splenomegaly Between the IDA Group and the Normal Population

\begin{tabular}{|c|l|c|c|c|}
\hline \multicolumn{2}{|c|}{} & \multicolumn{2}{c|}{ Results } & \multirow{2}{*}{ Total } \\
\cline { 3 - 4 } \multicolumn{2}{|c|}{} & $\begin{array}{c}\text { Non } \\
\text {-Splenomegaly }\end{array}$ & Splenomegaly & \\
\hline \multirow{2}{*}{ Group } & IDA & $622(93.1 \%)$ & $46(6.9 \%)$ & 668 \\
& Normal & $3180(99.3 \%)$ & $21(0.7 \%)$ & 3201 \\
\hline \multicolumn{2}{|c|}{ Total } & $3802(98.3 \%)$ & $67(1.7 \%)$ & 3869 \\
\hline
\end{tabular}

Notes: Comparison of the incidence of splenomegaly between the IDA group and the control group, the IDA group had a significantly higher incidence of splenomegaly than the control group. 
Table 2 Distribution of Splenomegaly Among Patients with Varying Degrees of IDA

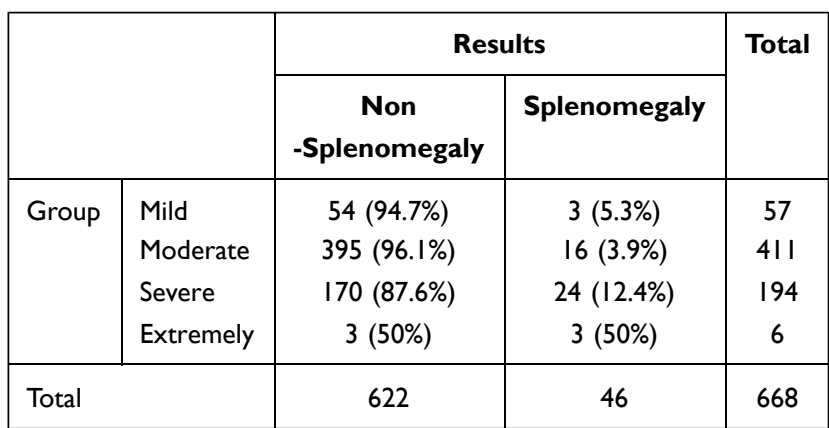

Notes: Comparison of the incidence of splenomegaly among patients with different degrees of IDA. The severe anemia group had a significantly higher incidence of splenomegaly than the mild anemia group and moderate anemia group.

infarction, and one died of heart disease), and eleven could not be contacted during follow-up. Among the 31 patients taking regular iron supplementation, abdominal Doppler ultrasound and abdominal palpation confirmed that the spleens of all patients were reduced by varying degrees. The spleens of 26 patients returned to a normal size, accounting for $83.9 \%$ of this subgroup. Among the 21 patients with splenomegaly in the control group, only 14 patients were followed up one year later, and the remaining seven patients refused further outpatient review due to personal reasons. Among the 14 patients, no spleens shrank.

\section{Discussion}

IDA is a common type of anemia seen in the Department of Hematology, and it comprises a series of disorders in which the production of hemoglobin is reduced due to a deficiency in iron, the raw material for hematopoiesis. ${ }^{1-3}$ Notably, the incidence is higher among women and children.

As an important immunological organ in the body, the spleen plays a key role in innate immunity and adaptive immunity. Additionally, one of its functions is the removal of aging and abnormal red blood cells. ${ }^{9-11}$ The many common causes of splenomegaly can be classified as being infectious or noninfectious. Infectious factors include viruses, bacteria, and parasites, and so on, whereas noninfectious factors include blood system disorders, connective tissue diseases, congestive splenomegaly, spleen tumors, and splenic cysts, among others. ${ }^{12}$

In this study, we found a relationship between IDA and splenomegaly, and this observation overturned the traditional cognition. Further analysis revealed a correlation between IDA severity and the likelihood of splenomegaly; that is, as the severity of IDA increased, the incidence of splenomegaly increased. The incidences of splenomegaly are low among patients with mild to moderate disease, only $5.3 \%$ and $3.9 \%$, respectively, but the incidence increases to $12.4 \%$ among patients with severe anemia and up to $50 \%$ among those with extremely severe anemia. However, splenomegaly disappears when the anemia is corrected. For the analysis of the possible causes of this phenomenon, we considered the possibility of extramedullary hematopoiesis. With the worsening of anemia, the degree of compensatory extramedullary hematopoiesis in the body increases, and the incidence of splenomegaly also increases. This hypothesis has been confirmed in recent studies. ${ }^{13}$ It is well-known that the bone marrow begins to demonstrate hematopoietic function at the 16th week of embryonic development when the hematopoietic function of the spleen and liver begins to gradually decline. ${ }^{14-16}$ In studies on the mechanism of hematopoiesis, hematopoiesis is inseparable from the microenvironment required by primitive cells for hematopoiesis. This explanation is also applicable to the occurrence of extramedullary hematopoiesis. Studies have found that the weight of the spleen in patients with extramedullary hematopoiesis is significantly higher than that in patients without extramedullary hematopoiesis, and the percentages of nonsegmental myeloid cells and CD71-positive cells in the spleens of patients with extramedullary hematopoiesis are significantly higher. ${ }^{17}$ This result has also been confirmed in animal models, ${ }^{13}$ and CD71 is generally considered a marker of cell proliferation. ${ }^{17}$ Another study showed that erythrocyte islands in the bone marrow are the basic structures of erythropoiesis. In the context of extramedullary hematopoiesis of the spleen, we observed the presence of erythrocytes, myeloid cells, and megakaryocytes in the intrasinus space of the spleen. In addition, erythrocyte islands were observed. ${ }^{17-20}$ Moreover, c-kit+CD34low/- cells have been shown to be part of hematopoietic precursor cells. The presence of such cells was also previously found in the spleen where extramedullary hematopoiesis occurred. ${ }^{18,20}$ Other reports have shown that bone marrow-derived hematopoietic stem cells (HSCs) were found in the sinuses of the spleen and near endothelial cells. $^{21-24}$ Therefore, we know that the presence of primitive cells and related functional structures can be observed during the process of extramedullary hematopoiesis of the spleen. In studies of the extramedullary hematopoietic microenvironment, we know that a variety of cytokines play a decisive role. Under pathological conditions, upregulation of bone marrow hematopoiesis leads to increased synthesis of growth factors for stem cell mobilization of chemokines and 
upregulation of hematopoietic function in embryonic hematopoietic sites (mainly the spleen and liver) or other tissues. ${ }^{4}$ However, in a mouse model with extramedullary hematopoiesis, a team found that conditional deletion of key niche factors, namely, SCF (also known as KITL) and CXCL12 from splenic endothelial cells, significantly reduced the occurrence of splenic extramedullary hematopoiesis and decreased the blood cell count without affecting bone marrow hematopoiesis. ${ }^{25,26}$ Further studies showed that CXCL12 expression significantly increased during extramedullary hematopoiesis, while splenic sinus endothelial cells expressing CXCL12 may attract hematopoietic precursor cells. ${ }^{17,24}$ At the same time, some studies have shown that hypoxia and other factors can also be used to stimulate extramedullary hematopoiesis in the body. ${ }^{21,27}$ Of course, this is only a tentative explanation of this clinical phenomenon. There is no direct evidence at present, and further studies with animal models are still needed.

Based on this study, we think that IDA can lead to the occurrence of splenomegaly to a certain extent and that the more serious the degree of IDA is, the higher the probability of splenomegaly will be. Compensatory extramedullary hematopoiesis of the spleen is considered to be caused by IDA. However, these are only our conjectures based on relevant studies. There is no direct evidence at present, and further animal experiments are needed.

\section{Data Sharing Statement}

Data sharing is not applicable to this article, as no datasets were generated or analyzed during the current study.

\section{Ethics Approval and Consent to Participate}

This study was approved by the Ethics Committee of Xinxiang Medical University affiliated with Puyang Oilfield General Hospital (Ethical review number: 201901-0003-E06). All participants provided informed consent to participate in this research and that it was conducted in accordance with the Declaration of Helsinki.

\section{Consent for Publication}

The authors obtained consent from the participants for publication of individual patient data.

\section{Acknowledgments}

We thank all the medical staff of our department for their help and guidance during the data collection.

\section{Author Contributions}

All authors made a significant contribution to the work reported, whether that is in the conception, study design, execution, acquisition of data, analysis and interpretation, or in all these areas; took part in drafting, revising or critically reviewing the article; gave final approval of the version to be published; have agreed on the journal to which the article has been submitted; and agree to be accountable for all aspects of the work.

\section{Funding}

There is no funding to report.

\section{Disclosure}

All the authors declare no conflicts of interest.

\section{References}

1. Kassebaum NJ, Jasrasaria R, Naghavi M, et al. A systematic analysis of global anemia burden from 1990 to 2010. Blood. 2014;123 (5):615-624. doi:10.1016/S0140-6736(16)31678-6

2. Lutter CK. Iron deficiency in young children in low-income countries and new approaches for its prevention. $J$ Nutr. 2008;138 (12):2523-2528. doi:10.3945/jn.108.095406

3. World Health Organization. Micronutrient Deficiencies. World Health Organization; 2014.

4. Kim Chang H. Homeostatic and pathogenic extramedullary hematopoiesis. J Blood Med. 2010;1:13-19. doi:10.2147/JBM.S7224

5. Groom AC, Eugene M. Landis award lecture microcirculation of the spleen: new concepts, new challenges. Microvasc Res. 1987;34 (3):269-289. doi:10.1016/0026-2862(87)90061-6

6. Chadburn A. The spleen: anatomy and anatomical function. Semin Hematol. 2000;37(1 Suppl 1):13-21. doi:10.1016/S0037-1963(00) 90113-6

7. Frank K, Linhart P, Kortsik C, et al. Sonographic determination of spleen size: normal dimensions in adults with a healthy spleen. Ultraschall in Der Medizin. 1986;7(3):134-137. doi:10.1016/j. ultrasmedbio.2006.06.017

8. Andrews MW. Ultrasound of the spleen. World J Surg. 2000;24 (2):183-187. doi:10.1007/s002689910031

9. Mebius RE, Kraal G. Structure and function of the spleen. Nat Rev Immunol. 2005;5(8):606-616. doi:10.1038/nri1669

10. Haan JMMD, Kraal G. Innate immune functions of macrophage subpopulations in the spleen. $J$ Innate Immun. 2012;4(56):437-445. doi:10.1159/000335216

11. Steiniger B, Barth P. Microanatomy and function of the spleen. $A d v$ Anat Embryol Cell Biol. 2000;151:III-IX, 1-101. doi:10.1007/9783-642-57088-9

12. Mckenzie CV, Colonne CK, Yeo JH, et al. Splenomegaly: pathophysiological bases and therapeutic options. Int J Biochem Cell Biol. 2018;94:40-43. doi:10.1016/j.biocel.2017.11.011

13. Li D, Peng J, Li T, et al. Itgb3-integrin-deficient mice may not be a sufficient model for patients with Glanzmann thrombasthenia. Mol Med Rep. 2021;23(6):6. doi:10.3892/mmr.2021.12088

14. Baron Margaret H. Embryonic origins of mammalian hematopoiesis. Exp Hematol. 2003;31(12):1160-1169. doi:10.1016/j. exphem.2003.08.019

15. Mikkola HKA, Gekas C, Orkin SH, et al. Placenta as a site for hematopoietic stem cell development. Exp Hematol. 2005;33 (9):1048-1054. doi:10.1016/j.exphem.2005.06.011 
16. McGrath K, Palis J. Ontogeny of erythropoiesis in the mammalian embryo. Curr Top Dev Biol. 2008;82(1):1-22. doi:10.1016/S00702153(07)00001-4

17. Miwa Y, Hayashi T, Suzuki S, et al. Up-regulated expression of CXCL12 in human spleens with extramedullary haematopoiesis. Pathology. $2013 ; 45(4): 408-416$

doi:10.1097/ PAT.0b013e3283613dbf

18. Sonoda Y. Immunophenotype and functional characteristics of human primitive CD34-negative hematopoietic stem cells: the significance of the intra-bone marrow injection. $J$ Autoimmun. 2008;30 (3):136-144. doi:10.1016/j.jaut.2007.12.004

19. Yoshida H, Kawane K, Koike M, et al. Phosphatidylserine-dependent engulfment by macrophages of nuclei from erythroid precursor cells. Nature. 2005;437(7059):754-758. doi:10.1038/nature03964

20. De Jong MO, Wagemaker G, Wognum AW. Separation of myeloid and erythroid progenitors based on expression of CD34 and c-kit. Blood. 1995;86(11):4076-4085. doi:10.1016/S0950-3536(05) 80254-9

21. Johns JL, Christopher MM. Extramedullary hematopoiesis: a new look at the underlying stem cell niche, theories of development, and occurrence in animals. Vet Pathol. 2012;49(3):508-523. doi:10.1177/ 0300985811432344
22. Asakura A, Rudnicki MA. Side population cells from diverse adult tissues are capable of in vitro hematopoietic differentiation. Exp Hematol. 2002;30(11):1339-1345. doi:10.1016/S0301-472X(02) 00954-2

23. Morita Y, Iseki A, Okamura S, et al. Functional characterization of hematopoietic stem cells in the spleen. Exp Hematol. 2011;39 (3):351-359.e3. doi:10.1016/j.exphem.2010.12.008

24. Yamamoto K, Miwa Y, Abe Suzuki S, et al. Extramedullary hematopoiesis: elucidating the function of the hematopoietic stem cell niche (review). Mol Med Rep. 2016;13(1):587-591. doi:10.3892/ mmr.2015.4621

25. Inra CN, Zhou BO, Acar M, et al. A perisinusoidal niche for extramedullary haematopoiesis in the spleen. Nature. 2015;527 (7579):466-471. doi:10.1038/nature15530

26. Ara T, Tokoyoda K, Sugiyama T, et al. Long-term hematopoietic stem cells require stromal cell-derived factor-1 for colonizing bone marrow during ontogeny. Immunity. 2003;19(2):257-267. doi:10.1016/S1074-7613(03)00201-2

27. Pak M, Lopez MA, Gabayan V, et al. Suppression of hepcidin during anemia requires erythropoietic activity. Blood. 2006;108 (12):3730-3735. doi:10.1182/blood-2006-06-028787
International Journal of General Medicine

\section{Publish your work in this journal}

The International Journal of General Medicine is an international, peer-reviewed open-access journal that focuses on general and internal medicine, pathogenesis, epidemiology, diagnosis, monitoring and treatment protocols. The journal is characterized by the rapid reporting of reviews, original research and clinical studies
Dovepress

across all disease areas. The manuscript management system is completely online and includes a very quick and fair peer-review system, which is all easy to use. Visit http://www.dovepress.com/ testimonials.php to read real quotes from published authors. 